\title{
Extremities Involving B. Y. Chen's Invariants for Real Hypersurfaces in Complex Quadric
}

\author{
Pooja Bansal* , Siraj Uddin and Mohammad Hasan Shahid \\ (Communicated by Ion Mihai)
}

\begin{abstract}
The article is concerned with the study of real hypersurfaces of the complex quadric $Q^{m}$. We establish B. Y. Chen's inequalities for real hypersurfaces of the complex quadric $Q^{m}$ and by considering the equality case, we obtain some consequences. Also, we establish an inequality in terms of the warping function and the scalar curvature for a warped product real hypersurface of $Q^{m}$ and some obstructions have been given. Moreover, we investigate the expression of the curvature tensor of a real hypersurface in the complex quadric $Q^{m}$ admitting semi-symmetric metric connection. Using this curvature, we derive inequalities involving Chen $\delta$-invariant admitting a semi-symmetric metric connection. Furthermore, the equality case is considered.
\end{abstract}

Keywords: real hypersurface; complex quadric; scalar curvature; Chen $\delta$-invariant; semi-symmetric metric connection.

AMS Subject Classification (2010): Primary: 53C40; Secondary: 53C55; 53B05; $53 B 15$.

\section{Introduction}

In 1968, S. S. Chern raised a question involving minimal isometric immersion into Euclidean space [12]. Then, Chen found some obstructions to Chern's problem and proposed inequalities for submanifolds in Riemannian space form concerning the sectional curvature, the scalar curvature and the squared mean curvature [9]. Moreover, he proposed inequality concerning $\delta\left(n_{1}, n_{2}, \ldots, n_{k}\right)$ and the squared mean curvature for the submanifolds in real space form [10].

Afterwards, many papers have been appeared in submanifolds of space forms in the version of real and complex like, generalised complex space forms [11], $(k, \mu)$-contact space forms [1] and Sasakian space forms [13]. Further, the geometry of the complex quadric has been studied by H. Reckziegel [16] in 1995 and Y. J. Suh, obtained some analyzing results on real hypersurfaces in the complex quadric by considering some geometric conditions like parallel Ricci tensor [17], Reeb parallel shape operator [18]. Also, the classifications of real hypersurface of the complex quadric with isometric Reeb flow were obtained by Berndt and Suh [5] and many more work have been studied by different authors considering the same ambient space ([2]-[4],[19]).

However, Hayden [14] originated the idea of a semi-symmetric metric connection on a Riemannian manifold. Yano [20] deliberated this connection and found some properties of a Riemannian manifold with the same connection. Also, A. Mihai and C. Özgür studied the Chen extremities for submanifolds of the real space forms with same connection [15].

Here, we first establish Chen's extremities for real hypersurfaces of the complex quadric $Q^{m}$ and considering the equality case, we obtain some consequences. Also, we establish an inequality in terms of the warping function and the scalar curvature for warped product real hypersurface of $Q^{m}$ and some obstructions have been given. Then, we study real hypersurface of $Q^{m}$ admitting semi-symmetric metric connection and find the curvature tensor of a real hypersurface in $Q^{m}$ with the semi-symmetric metric connection. Additionally, using this curvature we develop Chen's inequality for a real hypersurfaces of the complex quadric $Q^{m}$ admitting semi symmetric metric connection.

As long as, by virtue of simpleness, throughout a paper we denote semi-symmetric metric connection, LeviCivita connection and Warped product by SSMC, LC connection and WP, respectively. 


\section{The complex quadric $Q^{m}$}

For more details of the geometry of complex quadric we refer to ([5],[16],[17]). The complex hypersurface of $\mathbb{C} P^{m+1}$ is known as the complex quadric $Q^{m}$ defined by the equation $z_{1}^{2}+\ldots+z_{m+1}^{2}=0$, where $z_{1}, \ldots, z_{m+1}$ are homogeneous coordinates on $\mathbb{C} P^{m+1}$ equipped with the induced Riemannian metric $g$. Then, naturally the canonical Kähler structure $(J, g)$ on $Q^{m}$ is induced by Kähler structure on $\mathbb{C} P^{m+1}$ [18]. The 1-dimensional quadric $Q^{1}$ is congruent to the round 2-sphere $S^{2}$. The 2-dimensional quadric $Q^{2}$ is congruent to the Riemannian product $S^{2} \times S^{2}$. For this, we will assume $m \geq 3$ throughout the paper.

Apart from $J$ there is one more geometric structure on $Q^{m}$, known as the complex conjugation $A$ on the tangent spaces of $Q^{m}$ which is a parallel rank-two vector bundle $\mathcal{U}$ containing $S^{1}$-bundle of real structures. For $x \in Q^{m}$, let $A_{\bar{x}}$ be the shape operator of $Q^{m}$ in $\mathbb{C} P^{m+1}$. Then we have $A_{\bar{x}} W=W$ for $W \in T_{x} Q^{m}$, that is, $A$ is an involution or $A_{\bar{x}}$ is a complex conjugation restricted to $T_{x} Q^{m}$. Now, $T_{x} Q^{m}$ is decomposed as [18]:

$$
T_{x} Q^{m}=\mathcal{V}\left(A_{\bar{x}}\right) \oplus J \mathcal{V}\left(A_{\bar{x}}\right),
$$

such that $\mathcal{V}\left(A_{\bar{x}}\right)$ and $J \mathcal{V}\left(A_{\bar{x}}\right)$, respectively denote the (+1)-eigenspace and (-1)-eigenspace of the involution $A_{\bar{x}}^{2}=I$ on $T_{x} Q^{m}, x \in Q^{m}$.

Now, a tangent vector $W \neq 0 \in T_{x} Q^{m}$ is known as the singular if it is tangent to more than one maximal flat in $Q^{m}$. Classification of singular tangent vectors for $Q^{m}$ are given as [19]:

1 If there exists $A \in \mathcal{U}$ such that $W$ is an eigenvector corresponding to an eigenvalue (+1), then the singular tangent vector $W$ is known as $\mathcal{U}$-principal.

2 If there exists $A \in \mathcal{U}$ and orthonormal vectors $U, V \in \mathcal{V}(A)$ such that $W /\|W\|=(U+J V) / \sqrt{2}$, then the singular tangent vector $W$ is known as $\mathcal{U}$-isotropic.

Let $\mathcal{M}^{n}$ be a real hypersurface of $Q^{m}$ with a connection $\nabla$ induced from the LC connection $\bar{\nabla}$ in $Q^{m}$. Then, the transform $J U$ of the Kähler structure $J$ on $Q^{m}$ is defined by $J U=\phi U+\eta(U) N$ where $\phi U$ is the tangential component of $J U$ and $N \in T_{p}^{\perp} \mathcal{M}$, for $U \in T_{p} \mathcal{M}$. Here, $\mathcal{M}$ associates an induced almost contact metric structure $(\phi, \xi, \eta, g)$ satisfying the following relations [6]:

$$
\begin{array}{r}
\xi=-J N, \eta(\xi)=1, \eta(U)=g(\xi, U), \phi^{2} U+U=\eta(U) \xi, \phi \xi=0, \\
\eta(\phi U)=0, g(\phi U, \phi V)+\eta(U) \eta(V)=g(U, V), g(\phi U, V)=-g(U, \phi V) .
\end{array}
$$

Moreover, the real hypersurface $\mathcal{M}$ of $Q^{m}$ satisfy

$$
\nabla_{U} \xi=\phi S U
$$

where $S$ is the shaper operator of $\mathcal{M}$.

On the other hand, the Gauss and the Weingarten formulas for $M$ follows

$$
\bar{\nabla}_{U} V=\nabla_{U} V+h(U, V) \text { and } \bar{\nabla}_{U} N=-S U
$$

respectively, for $U, V \in T_{p} \mathcal{M}$ and $N \in T_{p}^{\perp} \mathcal{M}$. The second fundamental form $h$ and the shape operator $S$ of $\mathcal{M}$ are related by

$$
g(h(U, V), N)=g\left(S_{N} U, V\right)=g(S U, V) .
$$

Now, we take $A \in \mathcal{U}_{x}$ such that $N=\cos (t) Z_{1}+\sin (t) J Z_{2}$, where $Z_{1}, Z_{2}$ are orthonormal vectors in $\mathcal{V}(A)$ and $0 \leq t \leq \frac{\pi}{4}$ (see Proposition 3 [16]) which is a function on $\mathcal{M}$.

Since $\xi=-J N$, we have

$$
\begin{aligned}
N & =\cos (t) Z_{1}+\sin (t) J Z_{2}, \\
A N & =\cos (t) Z_{1}-\sin (t) J Z_{2}, \\
\xi & =\sin (t) Z_{2}-\cos (t) J Z_{1}, \\
A \xi & =\sin (t) Z_{2}+\cos (t) J Z_{1},
\end{aligned}
$$

from which it follows that $g(\xi, A N)=0$. 


\section{B. Y. Chen inequality for a real hypersurface of $Q^{m}$}

Here, we obtain the general inequality associated with the Chen $\delta$-invariant for a real hypersurfaces $\mathcal{M}$ of the complex quadric $Q^{m}$.

Now, from the Gauss equation, the Riemannian curvature tensor $R$ of connection $\nabla$ in terms of $J$ and $A \in \mathcal{U}$ is defined as [18]:

$$
\begin{aligned}
R(U, V) W= & g(V, W) U-g(U, W) V+g(\phi V, W) \phi U-g(\phi U, W) \phi V-2 g(\phi U, V) \phi W \\
& +g(A V, W) A U-g(A U, W) A V+g(J A V, W) J A U-g(J A U, W) J A V \\
& +g(S V, W) S U-g(S U, W) S V,
\end{aligned}
$$

where $U, V, W \in T_{p} \mathcal{M}$.

Then, we can see

$$
g\left(R(U, V) W+R(V, W) U+R(W, U) V, W^{\prime}\right)=0, \quad \text { for } U, V, W, W^{\prime} \in T_{p} M
$$

that is, the first Bianchi Identity holds for $\mathcal{M}$ of LC connection $\nabla$.

Next, the curvature tensor $R$ of the Hopf hypersurface $\mathcal{M}$ (i.e. $\alpha=g(S \xi, \xi)$ ), where $\alpha$ is a smooth function on $\mathcal{M}$ satisfies

$$
\begin{aligned}
R(U, \xi) V= & \eta(V)[U+\alpha S U]-[g(U, V)+\alpha g(S U, V)] \xi+g(A \xi, V) A U-g(A U, V) A \xi \\
& -g(A N, V) J A U+g(J A U, V) A N, \\
R(U, V) \xi= & \eta(V)[U+\alpha S U]-\eta(U)[V+\alpha S V]+g(A V, \xi) A U-g(A U, \xi) A V \\
& -g(A N, V) J A U+g(A U, N) J A V .
\end{aligned}
$$

Moreover, for a real hypersurface $\mathcal{M}$ and $U, V, W, W^{\prime} \in T_{p} \mathcal{M}$, the relation (3.1) produce

$$
\begin{aligned}
g\left(R(U, V) W, W^{\prime}\right)= & g(V, W) g\left(U, W^{\prime}\right)-g(U, W) g\left(V, W^{\prime}\right)+g(\phi V, W) g\left(\phi U, W^{\prime}\right) \\
& -g(\phi U, W) g\left(\phi V, W^{\prime}\right)-2 g(\phi U, V) g\left(\phi W, W^{\prime}\right)+g(A V, W) g\left(A U, W^{\prime}\right) \\
& -g(A U, W) g\left(A V, W^{\prime}\right)+g(J A V, W) g\left(J A U, W^{\prime}\right)-g(J A U, W) g\left(J A V, W^{\prime}\right) \\
& +g(S V, W) g\left(S U, W^{\prime}\right)-g(S U, W) g\left(S V, W^{\prime}\right) .
\end{aligned}
$$

By taking $U=W^{\prime}=e_{i}$ in (3.3), one can have [17]

$$
\begin{aligned}
\operatorname{Ric}(V, W)= & n g(V, W)-3 \eta(V) \eta(W)-g(A N, N) g(A V, W)+g(A W, N) g(A V, N) \\
& +g(A W, \xi) g(A V, \xi)+\operatorname{tr}(S) g(S V, W)-g\left(S^{2} V, W\right),
\end{aligned}
$$

where the Ricci tensor of $\mathcal{M}$ with connection $\nabla$ is symbolized by Ric which satisfy

$$
\operatorname{Ric}(U, \xi)=\left(2 n-4+\alpha h-\alpha^{2}\right) \eta(X)-2 g(A N, N) g(A U, \xi) .
$$

Consider an orthonormal basis $\left\{e_{i}\right\}_{1}^{n}$ and $\left\{e_{n+1}=N\right\}$ of $T_{p} \mathcal{M}$ and $T_{p}^{\perp} \mathcal{M}$ respectively, where $n+1=2 m$. Conveniently, let $h_{i j}^{n+1}=g\left(h\left(e_{i}, e_{j}\right), e_{n+1}\right)=g\left(h\left(e_{i}, e_{j}\right), N\right)$ for $i, j \in\{1, \ldots, n\}$. Now, one defines the squared mean curvature $\|\mathcal{H}\|^{2}$ of $\mathcal{M}$ in $Q^{m}$ and the squared norm $\|h\|^{2}$ of $h$ are given by:

$$
\|\mathcal{H}\|^{2}=\frac{1}{n^{2}}\left(\sum_{i, j=1}^{n} h_{i j}^{n+1}\right)^{2},\|h\|^{2}=\sum_{i, j=1}^{n}\left(h_{i j}^{n+1}\right)^{2},
$$

respectively.

Now, the scalar curvature $\tau$ has the expression

$$
\tau=\sum_{1 \leq i<j \leq n} \mathcal{K}\left(e_{i} \wedge e_{j}\right)
$$

where $\mathcal{K}(\pi)$ denotes the sectional curvature of $\mathcal{M}$ involved with a plane section $\pi \subset T_{p} \mathcal{M}$ and is spanned by tangent vectors $\left\{e_{i}, e_{j}\right\}$ and $\sum_{1<i<j<n} \mathcal{K}\left(e_{i} \wedge e_{j}\right)=\sum_{1 \leq i<j<n} g\left(R\left(e_{i}, e_{j}\right) e_{j}, e_{i}\right)$.

Revoke that the Chen first invariant ([9],[10]) is defined by

$$
\delta_{m}(p)=\tau(p)-\inf \left\{\mathcal{K}(\pi) \mid \pi \subset T_{p} \mathcal{M}, \operatorname{dim} \pi=2\right\},
$$

where $\tau(p)$ is the scalar curvature at $p$.

We give one algebraic result which we will use to proof our result. 
Lemma 3.1. [9] Let $a_{1}, a_{2}, \ldots, a_{k}, b$ be $(k+1)(k \geq 2)$ real numbers satisfying

$$
\left(\sum_{i=1}^{k} a_{i}\right)^{2}=(k-1)\left(\sum_{i=1}^{k} a_{i}^{2}+b\right)
$$

Then $2 a_{1} a_{2} \geq b$, with equality holding if and only if $a_{1}+a_{2}=a_{3}=\ldots=a_{k}$.

Theorem 3.1. For a real hypersurface $\mathcal{M}$ of $Q^{m}$ with 2-plane section $\pi \subset T_{p} \mathcal{M}$ spanned by tangent vectors $e_{1}$ and $e_{2}$, we have

$$
\tau(p)-\mathcal{K}(\pi) \leq \frac{n^{2}}{2}\left\{1+\left(\frac{n-2}{n-1}\right)\|\mathcal{H}\|^{2}\right\}+g^{2}\left(A e_{1}, e_{2}\right)+\frac{g^{2}(A N, N)}{2}+g^{2}\left(J A e_{1}, e_{2}\right) .
$$

Moreover, equality holds in (3.5) at $p \in \mathcal{M}$ if and only if there exist an orthonormal basis $\left\{e_{i}\right\}_{1}^{n}$ of $T_{p} \mathcal{M}$ and orthonormal normal frame $\left\{e_{n+1}=N\right\}$ of $T_{p}^{\perp} \mathcal{M}$, such that the matrix of the shape operator $S$ takes the following form

$$
S=\left(\begin{array}{ccc}
p^{\prime} & 0 & 0 \\
0 & q^{\prime} & 0 \\
0 & 0 & M
\end{array}\right)
$$

where $M$ is the diagonal matrix of order $n-2$ with diagonal entry $r=p^{\prime}+q^{\prime}$.

Proof. From (3.4), we deduce that

$$
2 \tau=n^{2}+g^{2}(A N, N)-1+n^{2}\|\mathcal{H}\|^{2}-\|h\|^{2}
$$

where we have used

$$
\begin{aligned}
\|h\|^{2} & =g\left(h\left(e_{i}, e_{j}\right), h\left(e_{i}, e_{j}\right)\right)=g\left(g\left(S e_{i}, e_{j}\right) N, g\left(S e_{i}, e_{j}\right) N\right) \\
& =\operatorname{tr}\left(S^{2}\right) .
\end{aligned}
$$

Let us denote

$$
\epsilon=2 \tau-n^{2}-g^{2}(A N, N)+1-\frac{n^{2}(n-2)}{n-1}\|\mathcal{H}\|^{2} .
$$

We obtain

$$
\epsilon=n^{2}\|\mathcal{H}\|^{2}-\|h\|^{2}-\frac{n^{2}(n-2)}{n-1}\|\mathcal{H}\|^{2}
$$

which provide

$$
n^{2}\|\mathcal{H}\|^{2}=(n-1)\left\{\epsilon+\|h\|^{2}\right\} .
$$

or, equivalently

$$
\left(\sum_{i=1}^{n} h_{i i}^{n+1}\right)^{2}=(n-1)\left\{\epsilon+\sum_{i=1}^{n}\left(h_{i j}^{n+1}\right)^{2}+\sum_{i \neq j}\left(h_{i j}^{n+1}\right)^{2}\right\} .
$$

Using lemma (3.1) together with equation (3.10), we obtain

$$
2 h_{11}^{n+1} h_{22}^{n+1} \geq \sum_{i \neq j}\left(h_{i j}^{n+1}\right)^{2}+\epsilon .
$$

Also, the Gauss equation implies that

$$
\begin{aligned}
\mathcal{K}(\pi)= & g\left(R\left(e_{1}, e_{2}\right) e_{2}, e_{1}\right) \\
= & 1+3 g^{2}\left(\phi e_{1}, e_{2}\right)+g\left(A e_{2}, e_{2}\right) g\left(A e_{1}, e_{1}\right)-g^{2}\left(A e_{1}, e_{2}\right) \\
& +g\left(J A e_{2}, e_{2}\right) g\left(J A e_{1}, e_{1}\right)-g^{2}\left(J A e_{1}, e_{2}\right)-\left(h_{12}^{n+1}\right)^{2}+h_{22}^{n+1} h_{11}^{n+1}
\end{aligned}
$$


Incorporating (3.11) in (3.12) yields

$$
\begin{aligned}
\mathcal{K}(\pi) \geq & 1+3 g^{2}\left(\phi e_{1}, e_{2}\right)+g\left(A e_{2}, e_{2}\right) g\left(A e_{1}, e_{1}\right)-g^{2}\left(A e_{1}, e_{2}\right)+g\left(J A e_{2}, e_{2}\right) g\left(J A e_{1}, e_{1}\right) \\
& -g^{2}\left(J A e_{1}, e_{2}\right)+\frac{1}{2}\left\{\sum_{i \neq j}\left(h_{i j}^{n+1}\right)^{2}+\epsilon\right\}-\left(h_{12}^{n+1}\right)^{2} \\
= & 1+3 g^{2}\left(\phi e_{1}, e_{2}\right)+g\left(A e_{2}, e_{2}\right) g\left(A e_{1}, e_{1}\right)-g^{2}\left(A e_{1}, e_{2}\right)+g\left(J A e_{2}, e_{2}\right) g\left(J A e_{1}, e_{1}\right) \\
& -g^{2}\left(J A e_{1}, e_{2}\right)+\tau-\frac{n^{2}}{2}-\frac{g^{2}(A N, N)}{2}+\frac{1}{2}-\frac{n^{2}(n-2)}{2(n-1)}\|\mathcal{H}\|^{2}+\frac{1}{2} \sum_{i \neq j, i, j \geq 2}\left(h_{i j}^{\alpha}\right)^{2} \\
\geq & 1+3 g^{2}\left(\phi e_{1}, e_{2}\right)+g\left(A e_{2}, e_{2}\right) g\left(A e_{1}, e_{1}\right)-g^{2}\left(A e_{1}, e_{2}\right)+g\left(J A e_{2}, e_{2}\right) g\left(J A e_{1}, e_{1}\right) \\
& -g^{2}\left(J A e_{1}, e_{2}\right)+\tau-\frac{n^{2}}{2}-\frac{g^{2}(A N, N)}{2}+\frac{1}{2}-\frac{n^{2}(n-2)}{2(n-1)}\|\mathcal{H}\|^{2} .
\end{aligned}
$$

Thus, finally we have

$$
\begin{aligned}
\tau(p)-\mathcal{K}(\pi) \leq & (n-2)\left\{\frac{n^{2}}{2(n-1)}\|\mathcal{H}\|^{2}+\frac{3 n-2}{2(n-2)}\right\}-3 g^{2}\left(\phi e_{1}, e_{2}\right)-g\left(A e_{2}, e_{2}\right) g\left(A e_{1}, e_{1}\right) \\
& +g^{2}\left(A e_{1}, e_{2}\right)-g\left(J A e_{2}, e_{2}\right) g\left(J A e_{1}, e_{1}\right)+g^{2}\left(J A e_{1}, e_{2}\right)+g\left(\phi S e_{2}, e_{2}\right) \\
& +g\left(\phi S e_{1}, e_{1}\right)-\eta\left(e_{2}\right)^{2}-\eta\left(e_{1}\right)^{2}+\frac{g^{2}(A N, N)}{2}+\frac{1}{2}-(n-1) g\left(\phi S e_{i}, e_{i}\right)
\end{aligned}
$$

or

$$
\tau(p)-\mathcal{K}(\pi) \leq \frac{n^{2}}{2}\left\{1+\left(\frac{n-2}{n-1}\right)\|\mathcal{H}\|^{2}\right\}+g^{2}\left(A e_{1}, e_{2}\right)+g^{2}\left(J A e_{1}, e_{2}\right)+\frac{g^{2}(A N, N)}{2} .
$$

Now, finally we get the equality in (13) at $p \in \mathcal{M}$ if and only if we have the equality case of lemma i.e.,

$$
\begin{aligned}
h_{i j}^{n+1} & =0 \quad \text { for all } i \neq j, \\
h_{11}^{n+1}+h_{22}^{n+1} & =h_{33}^{n+1}=h_{44}^{n+1}=\ldots=h_{n}^{n+1} .
\end{aligned}
$$

Thus, we may have the choice for $\left\{e_{1}, e_{2}\right\}$ such that $h_{12}^{n+1}=0$. Hence, the matrix of the shape operator has the form (3.6).

Corollary 3.1. Let $\mathcal{M}$ be a real hypersurface of $Q^{m}$ with 2-plane section $\pi \subset T_{p} \mathcal{M}$ spanned by tangent vectors $e_{1}$ and $e_{2}$ such that the normal vector field is $\mathcal{U}$-principal. Then, we have

$$
\tau(p)-\mathcal{K}(\pi) \leq \frac{n^{2}}{2}\left\{1+\left(\frac{n-2}{n-1}\right)\|\mathcal{H}\|^{2}\right\}+g^{2}\left(A e_{1}, e_{2}\right)+g^{2}\left(J A e_{1}, e_{2}\right)+\frac{1}{2} .
$$

Moreover, equality holds in (3.14) at $p \in \mathcal{M}$ if and only if there exist an orthonormal basis $\left\{e_{i}\right\}_{1}^{n}$ of $T_{p} \mathcal{M}$ and orthonormal normal frame $\left\{e_{n+1}=N\right\}$ of $T_{p}^{\perp} \mathcal{M}$, such that the matrix of the shape operator $S$ takes the following form

$$
S=\left(\begin{array}{ccc}
p^{\prime} & 0 & 0 \\
0 & q^{\prime} & 0 \\
0 & 0 & M
\end{array}\right),
$$

where $M$ is the diagonal matrix of order $n-2$ with diagonal entry $r=p^{\prime}+q^{\prime}$.

Corollary 3.2. Let $\mathcal{M}$ be a real hypersurface of $Q^{m}$ with 2-plane section $\pi \subset T_{p} \mathcal{M}$ spanned by tangent vectors $e_{1}$ and $e_{2}$ such that the normal vector field is $\mathcal{U}$-isotropic. Then, we have

$$
\tau(p)-\mathcal{K}(\pi) \leq \frac{n^{2}}{2}\left\{1+\left(\frac{n-2}{n-1}\right)\|\mathcal{H}\|^{2}\right\}+g^{2}\left(A e_{1}, e_{2}\right)+g^{2}\left(J A e_{1}, e_{2}\right) .
$$

Moreover, equality holds in (3.16) at $p \in \mathcal{M}$ if and only if there exist an orthonormal basis $\left\{e_{i}\right\}_{1}^{n}$ of $T_{p} \mathcal{M}$ and orthonormal normal frame $\left\{e_{n+1}=N\right\}$ of $T_{p}^{\perp} \mathcal{M}$, such that the matrix of the shape operator $S$ takes the following form

$$
S=\left(\begin{array}{ccc}
p^{\prime} & 0 & 0 \\
0 & q^{\prime} & 0 \\
0 & 0 & M
\end{array}\right),
$$

where $M$ is the diagonal matrix of order $n-2$ with diagonal entry $r=p^{\prime}+q^{\prime}$. 


\section{WP real hypersurface of $Q^{m}$}

In this section, we develop inequalities involving the warping function of a WP real hypersurface $\mathcal{M}$ of $Q^{m}$.

Next, we consider two Riemannian manifolds $\mathcal{M}_{1}$ and $\mathcal{M}_{2}$ of dimensions $n_{1}$ and $n_{2}$ equipped with Riemannian metrics $\varsigma_{1}$ and $\varsigma_{2}$ respectively. Let $\zeta$ be a positive function on $\mathcal{M}_{1}$. The WP manifold $\mathcal{M}_{1} \otimes_{\zeta} \mathcal{M}_{2}$ is defined to be the product manifold $\mathcal{M}_{1} \otimes \mathcal{M}_{2}$ with the warped metric $g=\varsigma_{1}+\zeta^{2} \varsigma_{2}$ [7].

Consider an isometric immersion $\Psi: \mathcal{M}=\mathcal{M}_{1} \otimes_{\zeta} \mathcal{M}_{2} \rightarrow Q^{m}$ of a WP manifold $\mathcal{M}_{1} \otimes_{\zeta} \mathcal{M}_{2}$ into a Riemannian manifold $Q^{m}$. Let $h$ be the second fundamental form of $\Psi$ and the mean curvature vectors denoted by $\mathcal{H}_{i}=\frac{1}{n_{i}} \operatorname{tr}\left(h_{i}\right)$ where $\operatorname{tr}\left(h_{i}\right)$ is the trace of $h$ restricted to $\mathcal{M}_{i}(i=1,2)$.

Theorem 4.1. Let $\Psi: \mathcal{M}^{n}=\mathcal{M}_{1} \otimes_{\zeta} \mathcal{M}_{2} \rightarrow Q^{m}$ be an isometric immersion of a WP real hypersurface into $Q^{m}$ with $\xi \in T_{p} \mathcal{M}_{1}$. Then

$$
n_{2} \frac{\Delta \zeta}{\zeta} \leq-\frac{n^{2}}{2}+\frac{1}{2} g^{2}(A N, N)-2 n+2 n_{1} n_{2}+\frac{11}{2}+\frac{n^{2}}{4}\|\mathcal{H}\|^{2}+2 \sum_{i=1}^{n} g\left(A^{2} e_{i}, e_{i}\right),
$$

where $n_{i}=\operatorname{dim} \mathcal{M}_{i}$ for $i=1,2, \Delta$ is the Laplacian operator of $\mathcal{M}_{1}$ and $\left\{e_{i}\right\}_{1}^{n}$ is an orthonormal basis of $T_{p} \mathcal{M}$.

Proof. Let us consider an isometric immersion $\Psi: \mathcal{M}=\mathcal{M}_{1} \otimes_{\zeta} \mathcal{M}_{2} \rightarrow \mathcal{N}(s)$ of a WP real hypersurface $\mathcal{M}_{1} \otimes_{\zeta}$ $\mathcal{M}_{2}$ into $Q^{m}$ whose structure vector field $\xi \in T_{p} \mathcal{M}_{1}$. Then, one can easily have [8]

$$
\mathcal{K}(X \wedge Z)=\frac{1}{\zeta}\left\{\left(\nabla_{X} X\right) \zeta-X^{2} \zeta\right\} .
$$

Now we choose an orthonormal basis $\left\{e_{i}\right\}_{1}^{n}$ of $T_{p} \mathcal{M}$ such that $e_{1}, \ldots, e_{n_{1}}$ are tangent to $\mathcal{M}_{1}$ and $e_{n_{1}+1}, \ldots, e_{n}$ are tangent to $\mathcal{M}_{2}$. Then, with the virtue of above defined relation, we obtain

$$
\frac{\Delta \zeta}{\zeta}=\sum_{1 \leq i \leq n_{1}} \sum_{n_{1}+1 \leq j \leq n} \mathcal{K}\left(e_{i} \wedge e_{j}\right)
$$

By definition of scalar curvture $\tau$ and (4.1) yields

$$
n_{2} \frac{\Delta \zeta}{\zeta}=\tau-\sum_{1 \leq i \leq n_{1}} \mathcal{K}\left(e_{i} \wedge e_{j}\right)-\sum_{n_{1}+1 \leq j \leq n} \mathcal{K}\left(e_{i} \wedge e_{j}\right)
$$

From (3.7), we have

$$
n^{2}\|\mathcal{H}\|^{2}=2\left(\delta+\|h\|^{2}\right)
$$

where

$$
\delta=2 \tau-n^{2}-g^{2}(A N, N)+1-\frac{n^{2}}{2}\|\mathcal{H}\|^{2} .
$$

Moreover, in local coordinates (4.3) has the following expression

$$
\left(\sum_{i=1}^{n} h_{i i}^{n+1}\right)^{2}=2\left(\delta+\sum_{i=1}^{n}\left(h_{i i}^{n+1}\right)^{2}+\sum_{i \neq j}\left(h_{i i}^{n+1}\right)^{2}\right)
$$

or, equivalently

$$
\begin{aligned}
\left(h_{11}^{n+1}+\sum_{i=2}^{n_{1}} h_{i i}^{n+1}+\sum_{i=n_{1}+1}^{n} h_{i i}^{n+1}\right)^{2}= & 2\left\{\delta+\left(h_{11}^{n+1}\right)^{2}+\sum_{i=2}^{n_{1}}\left(h_{i i}^{n+1}\right)^{2}+\sum_{i=n_{1}+1}^{n}\left(h_{i i}^{n+1}\right)^{2}\right. \\
& \left.+\sum_{1 \leq i \neq j \leq n}\left(h_{i j}^{n+1}\right)^{2}\right\} \\
= & 2\left\{\delta+\left(h_{11}^{n+1}\right)^{2}+\left(\sum_{i=2}^{n_{1}} h_{i i}^{n+1}\right)^{2}-\sum_{2 \leq j \neq k \leq n_{1}} h_{j j}^{n_{1}+1} h_{k k}^{n_{1}+1}\right. \\
& \left.+\left(\sum_{i=n_{1}+1}^{n} h_{i i}^{n+1}\right)^{2}-\sum_{n_{1}+1 \leq j \neq k \leq n} h_{j j} h_{k k}+\sum_{1 \leq i \neq j \leq n}\left(h_{i j}^{n+1}\right)^{2}\right\}
\end{aligned}
$$


Using lemma (3.1), we have

$$
\sum_{1 \leq j \neq k \leq n_{1}} h_{j j}^{n+1} h_{k k}^{n+1}+\sum_{n_{1}+1 \leq j \neq k \leq n} h_{j j}^{n+1} h_{k k}^{n+1} \geq \frac{\delta}{2}+\sum_{1 \leq i<j \leq n}\left(h_{i j}^{n+1}\right)^{2}
$$

Furthermore, equality holds if and only if

$$
\sum_{i=1}^{n_{1}} h_{i i}^{n+1}=\sum_{i=n_{1}+1}^{n} h_{i i}^{n+1}
$$

We also know that

$$
\begin{aligned}
\tau & =\sum_{1 \leq i<j \leq n} \mathcal{K}\left(e_{i} \wedge e_{j}\right) \\
& =\sum_{1 \leq i<j \leq n_{1}} \mathcal{K}\left(e_{i} \wedge e_{j}\right)+\sum_{n_{1}+1 \leq i<j \leq n} \mathcal{K}\left(e_{i} \wedge e_{j}\right)+\sum_{j=n_{1}+1}^{n} \sum_{i=1}^{n_{1}} \mathcal{K}\left(e_{i} \wedge e_{j}\right)
\end{aligned}
$$

So, from (4.1), we derive

$$
\begin{aligned}
n_{2} \frac{\Delta \zeta}{\zeta}= & \sum_{j=n_{1}+1}^{n} \sum_{i=1}^{n_{1}} \mathcal{K}\left(e_{i} \wedge e_{j}\right) \\
= & \tau-\sum_{1 \leq i<j \leq n_{1}} \mathcal{K}\left(e_{i} \wedge e_{j}\right)-\sum_{n_{1}+1 \leq i<j \leq n} \mathcal{K}\left(e_{i} \wedge e_{j}\right) \\
= & \tau-n_{1}\left(n_{1}-1\right)-3\left(n_{1}-1\right)-n_{2}\left(n_{2}-1\right)-3\left(n_{2}-1\right)+2 \sum_{i=1}^{n} g\left(A^{2} e_{i}, e_{i}\right) \\
& -\left\{\sum_{1 \leq i<j \leq n_{1}} g\left(A e_{i}, e_{i}\right) g\left(A e_{j}, e_{j}\right)+\sum_{n_{1}+1 \leq i<j \leq n} g\left(A e_{i}, e_{i}\right) g\left(A e_{j}, e_{j}\right)\right\} \\
& -\left\{\sum_{1 \leq i<j \leq n_{1}} g\left(J A e_{i}, e_{i}\right) g\left(J A e_{j}, e_{j}\right)+\sum_{n_{1}+1 \leq i<j \leq n} g\left(J A e_{i}, e_{i}\right) g\left(J A e_{j}, e_{j}\right)\right\} \\
& -\left\{\sum_{1 \leq i<j \leq n_{1}} h_{i i}^{n+1} h_{j j}^{n+1}+\sum_{n_{1}+1 \leq i<j \leq n} h_{i i}^{n+1} h_{j j}^{n+1}\right\}+\left\{\sum_{1 \leq i<j \leq n_{1}}\left(h_{i j}^{n+1}\right)^{2}\right. \\
& \left.+\sum_{n_{1}+1 \leq i<j \leq n}\left(h_{i j}^{n+1}\right)^{2}\right\}
\end{aligned}
$$

Using (4.5), we have

$$
\begin{aligned}
n_{2} \frac{\Delta \zeta}{\zeta} \leq & \tau-n_{1}\left(n_{1}-1\right)-3\left(n_{1}-1\right)-n_{2}\left(n_{2}-1\right)-3\left(n_{2}-1\right)+2 \sum_{i=1}^{n} g\left(A^{2} e_{i}, e_{i}\right) \\
& -\left\{\sum_{1 \leq i<j \leq n_{1}} g\left(A e_{i}, e_{i}\right) g\left(A e_{j}, e_{j}\right)+\sum_{n_{1}+1 \leq i<j \leq n} g\left(A e_{i}, e_{i}\right) g\left(A e_{j}, e_{j}\right)\right\} \\
& -\left\{\sum_{1 \leq i<j \leq n_{1}} g\left(J A e_{i}, e_{i}\right) g\left(J A e_{j}, e_{j}\right)+\sum_{n_{1}+1 \leq i<j \leq n} g\left(J A e_{i}, e_{i}\right) g\left(J A e_{j}, e_{j}\right)\right\} \\
& -\left\{\frac{1}{2} \delta+\sum_{1 \leq i<j \leq n_{1}}\left(h_{i j}^{n+1}\right)^{2}\right\}+\left\{\sum_{1 \leq i<j \leq n_{1}}\left(h_{i j}^{n+1}\right)^{2}+\sum_{n_{1}+1 \leq i<j \leq n}\left(h_{i j}^{n+1}\right)^{2}\right\}
\end{aligned}
$$

which gives

$$
n_{2} \frac{\Delta \zeta}{\zeta} \leq \tau-n_{1}\left(n_{1}-1\right)-3\left(n_{1}-1\right)-n_{2}\left(n_{2}-1\right)-3\left(n_{2}-1\right)+2 \sum_{i=1}^{n} g\left(A^{2} e_{i}, e_{i}\right)-\frac{1}{2} \delta .
$$


Incorporating (4.4) with the above relation, we derive

$$
n_{2} \frac{\Delta \zeta}{\zeta} \leq-\frac{1}{2} n^{2}+\frac{1}{2} g^{2}(A N, N)-2 n+2 n_{1} n_{2}+\frac{11}{2}+\frac{n^{2}}{4}\|\mathcal{H}\|^{2}+2 \sum_{i=1}^{n} g\left(A^{2} e_{i}, e_{i}\right)-\frac{1}{2} \delta
$$

from which we conclude our result.

Corollary 4.1. Let $\Psi: \mathcal{M}^{n}=\mathcal{M}_{1} \otimes_{\zeta} \mathcal{M}_{2} \rightarrow Q^{m}$ be an isometric immersion of a WP real hypersurface into $Q^{m}$ with $\xi \in T_{p} \mathcal{M}_{1}$. Then, for a $\mathcal{U}$-principal normal vector field, we have the inequality

$$
n_{2} \frac{\Delta \zeta}{\zeta} \leq-\frac{n^{2}}{2}-2 n+2 n_{1} n_{2}+6+\frac{n^{2}}{4}\|\mathcal{H}\|^{2}+2 \sum_{i=1}^{n} g\left(A^{2} e_{i}, e_{i}\right)
$$

where $n_{i}=\operatorname{dim} \mathcal{M}_{i}$, for $i=1,2, \Delta$ is the Laplacian operator of $\mathcal{M}_{1}$ and $\left\{e_{i}\right\}_{1}^{n}$ is an orthonormal basis of $T_{p} \mathcal{M}$.

Corollary 4.2. Let $\Psi: \mathcal{M}^{n}=\mathcal{M}_{1} \otimes_{\zeta} \mathcal{M}_{2} \rightarrow Q^{m}$ be an isometric immersion of a WP real hypersurface into $Q^{m}$ with $\xi \in T_{p} \mathcal{M}_{1}$. Then, for a $\mathcal{U}$-isotropic normal vector field, we have the inequality

$$
n_{2} \frac{\Delta \zeta}{\zeta} \leq-\frac{n^{2}}{2}-2 n+2 n_{1} n_{2}+\frac{11}{2}+\frac{n^{2}}{4}\|\mathcal{H}\|^{2}+2 \sum_{i=1}^{n} g\left(A^{2} e_{i}, e_{i}\right)
$$

where $n_{i}=\operatorname{dim} \mathcal{M}_{i}$, for $i=1,2, \Delta$ is the Laplacian operator of $\mathcal{M}_{1}$ and $\left\{e_{i}\right\}_{1}^{n}$ is an orthonormal basis of $T_{p} \mathcal{M}$.

\section{Curvature tensor of real hypersurface $\mathcal{M}$ in $Q^{m}$ admitting SSMC}

In this section, we study SSMC and then we obtain the curvature tensor of a real hypersurface $\mathcal{M}$ in $Q^{m}$ with respect to SSMC and then we find the intrinsic scalar curvature with respect to SSMC.

Consider a Riemannian manifold $\left(\mathcal{M}^{n}, g\right)$ with linear connection $\tilde{\nabla}$. Then, $\tilde{\nabla}$ is called semi-symmetric connection [20] if its torsion tensor $\tilde{\mathcal{T}}$, defined by

$$
\tilde{\mathcal{T}}(U, V)=\tilde{\nabla}_{U} V-\tilde{\nabla}_{V} U-[U, V],
$$

satisfy

$$
\tilde{\mathcal{T}}(U, V)=\eta(V) U-\eta(U) V,
$$

for $U, V \in T_{p} \mathcal{M}$ and a 1-form $\eta$. In addition, a semi-symmetric linear connection is said to be SSMC $\tilde{\nabla}$ if it holds

$$
\tilde{\nabla} g=0,
$$

for all $U, V \in T_{p} \mathcal{M}$, otherwise it is said to be a semi-symmetric non-metric connection.

A SSMC $\tilde{\nabla}$ in terms of the LC connection $\nabla$ on $\mathcal{M}$ is defined by

$$
\tilde{\nabla}_{U} V=\nabla_{U} V+\eta(V) U-g(U, V) \xi
$$

for $U, V \in T_{p} \mathcal{M}$.

Now, let us consider the complex quadric $Q^{m}$ admitting SSMC $\tilde{\nabla}$ and the LC connection $\bar{\nabla}$. Next, let $\mathcal{M}$ be a real hypersurface of $Q^{m}$ with the induced SSMC $\tilde{\nabla}$ and the induced LC connection $\nabla$. Let $\tilde{\bar{R}}$ and $\bar{R}$ be the curvature tensors of $Q^{m}$ with respect to the connections $\tilde{\nabla}$ and $\bar{\nabla}$ respectively. Put $\tilde{R}$ as the curvature tensor field of $\tilde{\nabla}$ and $R$ as the curvature tensor field of $\nabla$ on $\mathcal{M}$. Then the Gauss formulae with respect to $\tilde{\nabla}$ and $\nabla$ has the expression

$$
\tilde{\bar{\nabla}}_{U} V=\tilde{\nabla}_{U} V+\tilde{h}(U, V), \quad \bar{\nabla}_{U} V=\nabla_{U} V+h(U, V)
$$

respectively, where $\tilde{h}$ is the (0,2)-tensor of $\mathcal{M}$ in $Q^{m}$ and from these two relations, one can easily get $\tilde{h}(U, V)=$ $h(U, V)$. 
Furthermore, using (5.4) for $U, V \in T_{p} \mathcal{M}$, we have

$$
\begin{aligned}
\left(\tilde{\nabla}_{U} \eta\right)(V) & =\left(\nabla_{U} \eta\right)(V)+g(\phi U, \phi V)=g(\phi S U, V)+g(\phi U, \phi V), \\
\left(\tilde{\nabla}_{U} \phi\right)(V) & =\left(\nabla_{U} \phi\right)(V)-g(U, \phi V) \xi-\eta(V) \phi U \\
& =\eta(V) S U-\eta(V) \phi U-g(S U, V) \xi+g(\phi U, V) \xi,
\end{aligned}
$$

and the covariant derivative of torsion tensor of $\tilde{\nabla}$ with respect to SSMC follows

$$
\begin{aligned}
\left(\tilde{\nabla}_{U} \tilde{\mathcal{T}}\right)(V, W)= & g(\phi S U, V) W-g(\phi S U, W) V+g(U, V) W-g(U, W) V \\
& -\eta(U)[\eta(V) W-\eta(W) V]
\end{aligned}
$$

for $U, V, W \in T_{p} \mathcal{M}$.

Now, we know the curvature tensor $\tilde{R}$ can be calculated by

$$
\tilde{R}(U, V) W=\tilde{\nabla}_{U} \tilde{\nabla}_{V} W-\tilde{\nabla}_{V} \tilde{\nabla}_{U} W-\tilde{\nabla}_{[U, V]} W .
$$

Thus, using the relation (5.4), we obtain the relation between curvature tensor vector $\tilde{R}$ and $R$ of $\mathcal{M}$ in $Q^{m}$ admitting SSMC $\tilde{\nabla}$ and LC connection $\nabla$ given by

$$
\begin{aligned}
\tilde{R}(U, V) W= & R(U, V) W+g(\phi S U, W) V-g(\phi S V, W) U+\eta(W)[\eta(V) U-\eta(U) V] \\
& -g(V, W)[\phi S U+U-\eta(U) \xi]+g(U, W)[\phi S V+V-\eta(V) \xi]
\end{aligned}
$$

Then from (5.5), one can easily obtain

$$
\begin{aligned}
\tilde{R}(U, \xi) W & =R(U, \xi) W+g(\phi S U, W) \xi-\eta(W) \phi S U \\
\tilde{R}(U, V) \xi & =R(U, V) \xi-\eta(V) \phi S U+\eta(U) \phi S V .
\end{aligned}
$$

Also for $U, V, W, W^{\prime} \in T_{p} \mathcal{M}$, we have

$$
\begin{gathered}
g\left(\tilde{R}(V, U) W, W^{\prime}\right)=-g\left(\tilde{R}(U, V) W, W^{\prime}\right), \\
g\left(\tilde{R}(U, V) W^{\prime}, W\right)=-g\left(\tilde{R}(U, V) W, W^{\prime}\right)
\end{gathered}
$$

Now, if we assume that $\mathcal{M}$ satisfies $\phi S+S \phi=0$, then we derive

$$
\begin{array}{r}
g\left(\tilde{R}\left(W, W^{\prime}\right) U, V\right)=g\left(\tilde{R}(U, V) W, W^{\prime}\right), \\
g\left(\tilde{R}(U, V) W+\tilde{R}(V, W) U+\tilde{R}(W, U) V, W^{\prime}\right)=0 .
\end{array}
$$

Thus, we are able to state the following results

Theorem 5.1. Let $M$ be a real hypersurface $\mathcal{M}$ in $Q^{m}$ admitting $S S M C$. Then for $U, V, W, W^{\prime} \in T_{p} \mathcal{M}$, we have

(a) The curvature tensor of $\mathcal{M}$ with SSMC is given by (5.5)

(b) $g\left(\tilde{R}(V, U) W, W^{\prime}\right)+g\left(\tilde{R}(U, V) W, W^{\prime}\right)=0$

(c) $g\left(\tilde{R}(U, V) W^{\prime}, W\right)+g\left(\tilde{R}(U, V) W, W^{\prime}\right)=0$.

Proposition 5.1. In a real hypersurface $\mathcal{M}$ of $Q^{m}$ admitting $S S M C$ together with $\phi S+S \phi=0$, we have

(a) $g\left(\tilde{R}(U, V) W, W^{\prime}\right)-g\left(\tilde{R}\left(W, W^{\prime}\right) U, V\right)=0$ for $U, V, W, W^{\prime} \in T_{p} \mathcal{M}$

(b) $\mathcal{M}$ holds first Bianchi identity with respect to SSMC.

Proof. By using the assumption, the result follows immediately.

Relation (5.5) can be rewritten as

$$
\begin{aligned}
g\left(\tilde{R}(U, V) W, W^{\prime}\right)= & g\left(R(U, V) W, W^{\prime}\right)+\eta(U)\left[\eta(W) g\left(S V, W^{\prime}\right)-g(S V, W) \eta\left(W^{\prime}\right)\right] \\
& -\eta(V)\left[\eta(W) g\left(S U, W^{\prime}\right)-g(S U, W) \eta\left(W^{\prime}\right)\right]-g(\phi S U, V) g\left(\phi W, W^{\prime}\right) \\
& +g(\phi S V, U) g\left(\phi W, W^{\prime}\right) .
\end{aligned}
$$


Now, on contracting $U$ and $W^{\prime}$ in above defined relation, we derive

$$
\begin{aligned}
\tilde{\operatorname{Ric}}(V, W)= & \operatorname{Ric}(V, W)-(n-2) g(\phi S V, W)+(n-2) \eta(V) \eta(W) \\
& -g(V, W)\left[\sum_{i=1}^{n} g\left(\phi S e_{i}, e_{i}\right)+(n-2)\right],
\end{aligned}
$$

where $\tilde{\operatorname{Ric}}(V, W)$ and $\operatorname{Ric}(V, W)$ are the Ricci tensors of the connection $\tilde{\nabla}$ and $\nabla$ respectively.

Again, by applying contraction on $V$ and $W$, the scalar curvature $\tilde{\tau}$ with SSMC has the following expression

$$
2 \tilde{\tau}=3(n-1)-2+g^{2}(A N, N)+n^{2}\|\tilde{\mathcal{H}}\|^{2}-\|h\|^{2}-2(n-1) g\left(\phi S e_{i}, e_{i}\right) .
$$

Thus, we have

Lemma 5.1. In a real hypersurface $\mathcal{M}$ of $Q^{m}$ admitting $S S M C$ such that $\phi S=S \phi$, we have

(a) $\tilde{\operatorname{Ric}}(V, W)=\operatorname{Ric}(V, W)-(n-2)[g(\phi S V, W)+g(\phi V, \phi W)]$

(b) $\tilde{Q} V=Q V-(n-2)[V-\eta(V) \xi-\phi S V]$

(c) $\operatorname{Ric}(V, \xi)$ coincides with $\operatorname{Ric}(V, \xi)$

for all $V, W \in T_{p} \mathcal{M}$.

Proof. Let us assume that $\phi S=S \phi$. Then, we have

$$
\begin{aligned}
g\left(\phi S e_{i}, e_{i}\right) & =g\left(S \phi e_{i}, e_{i}\right) \\
& =-g\left(\phi S e_{i}, e_{i}\right)
\end{aligned}
$$

This results $g\left(\phi S e_{i}, e_{i}\right)=0$, which together with (5.6) follows (a) and hence (b). By using the assumption and inserting $W=\xi$ in (5.6), we get (c).

Also, we know that the Ricci operator $\tilde{Q}$ of SSMC is defined by

$$
\tilde{\operatorname{Ric}}(V, W)=g(\tilde{Q} V, W), \forall V, W \in T_{p} \mathcal{M} .
$$

From this incorporating (5.6) together with the assumption, we have

$$
\tilde{Q} V=Q V-(n-2)[V-\eta(V) \xi-\phi S V] .
$$

\section{Chen's inequality for a real hypersurface $\mathcal{M}$ of $Q^{m}$ with SSMC}

Here, we obtain inequality for the mean curvature, the scalar and the sectional curvature associated with the induced SSMC for a real hypersurfaces $\mathcal{M}$ of $Q^{m}$.

Here, we have the squared mean curvature $\|\tilde{\mathcal{H}}\|^{2}$ of $M$ in $Q^{M}$ and the squared norm $\|h\|^{2}$ of $h$ as

$$
\|\tilde{\mathcal{H}}\|^{2}=\frac{1}{n^{2}}\left(\sum_{i, j=1}^{n} h_{i j}^{n+1}\right)^{2} \text { and }\|h\|^{2}=\sum_{i, j=1}^{n}\left(h_{i j}^{n+1}\right)^{2}
$$

respectively, where $h_{i j}^{n+1}=g\left(h\left(e_{i}, e_{j}\right), N\right)$ and the mean curvature vector field $\tilde{\mathcal{H}}$ of $\tilde{\nabla}$ and $\mathcal{H}$ of $\nabla$ are invariant. Now, the scalar curvature $\tilde{\tau}$ for an orthonormal basis $\left\{e_{i}\right\}_{1}^{n}$ reads

$$
\tilde{\tau}=\sum_{1 \leq i<j \leq n} \mathcal{K}\left(e_{i} \wedge e_{j}\right)
$$

Theorem 6.1. Let $\mathcal{M}$ be a real hypersurface of $Q^{m}$ admitting SSMC $\tilde{\nabla}$. Then, for 2-plane section $\pi \subset T_{p} \mathcal{M}$ spanned by tangent vectors $e_{1}$ and $e_{2}$, we have

$$
\begin{aligned}
\tilde{\tau}(x)-\mathcal{K}(\pi) \leq & (n-2)\left\{\frac{n^{2}\|\tilde{\mathcal{H}}\|^{2}}{2(n-1)}+\frac{3(n-1)-2}{2(n-2)}\right\}+g^{2}\left(A e_{1}, e_{2}\right)+\frac{g^{2}(A N, N)}{2} \\
& +g\left(\phi S e_{1}, e_{1}\right)+g\left(\phi S e_{2}, e_{2}\right)+g^{2}\left(J A e_{1}, e_{2}\right)+(n-1) g\left(\phi S e_{i}, e_{i}\right) .
\end{aligned}
$$


Moreover, equality holds in (6.1) at $p \in \mathcal{M}$ if and only if there exist an orthonormal basis $\left\{e_{i}\right\}_{1}^{n}$ of $T_{p} \mathcal{M}$ and orthonormal normal frame $\left\{e_{n+1}=N\right\}$ of $T_{p}^{\perp} \mathcal{M}$, such that the matrix of the shape operator $S$ takes the following form

$$
S=\left(\begin{array}{ccc}
p^{\prime} & 0 & 0 \\
0 & q^{\prime} & 0 \\
0 & 0 & M
\end{array}\right),
$$

where $M$ is the diagonal matrix of oerder $n-2$ with diagonal entry $r=p^{\prime}+q^{\prime}$

Proof. First of all we put

$$
\epsilon=2 \tilde{\tau}-3(n-1)+2-g^{2}(A N, N)+2(n-1) g\left(\phi S e_{i}, e_{i}\right)-\frac{n^{2}(n-2)}{n-1}\|\tilde{\mathcal{H}}\|^{2}
$$

Thus, we have

$$
n^{2}\|\tilde{\mathcal{H}}\|^{2}=(n-1)\left\{\epsilon+\|h\|^{2}\right\} .
$$

Moreover, we can write

$$
\left(\sum_{i=1}^{n} h_{i i}^{n+1}\right)^{2}=(n-1)\left\{\epsilon+\sum_{i=1}^{n}\left(h_{i j}^{n+1}\right)^{2}+\sum_{i \neq j}\left(h_{i j}^{n+1}\right)^{2}\right\}
$$

Using lemma (3.1), we obtain

$$
2 h_{11}^{n+1} h_{22}^{n+1} \geq \sum_{i \neq j}\left(h_{i j}^{n+1}\right)^{2}+\epsilon
$$

The Gauss equation yields

$$
\begin{aligned}
\tilde{\mathcal{K}}(\pi)= & g\left(\tilde{R}\left(e_{1}, e_{2}\right) e_{2}, e_{1}\right) \\
= & 3 g^{2}\left(\phi e_{1}, e_{2}\right)+g\left(A e_{2}, e_{2}\right) g\left(A e_{1}, e_{1}\right)-g^{2}\left(A e_{1}, e_{2}\right)+g\left(J A e_{2}, e_{2}\right) g\left(J A e_{1}, e_{1}\right)-\left(h_{12}^{n+1}\right)^{2} \\
& +h_{22}^{n+1} h_{11}^{n+1}-g\left(\phi S e_{2}, e_{2}\right)-g\left(\phi S e_{1}, e_{1}\right)+\eta\left(e_{2}\right)^{2}+\eta\left(e_{1}\right)^{2}-g^{2}\left(J A e_{1}, e_{2}\right)
\end{aligned}
$$

Inserting (6.6) into (6.7) yields

$$
\begin{aligned}
\tilde{\mathcal{K}}(\pi) \geq & 3 g^{2}\left(\phi e_{1}, e_{2}\right)+g\left(A e_{2}, e_{2}\right) g\left(A e_{1}, e_{1}\right)-g^{2}\left(A e_{1}, e_{2}\right)+g\left(J A e_{2}, e_{2}\right) g\left(J A e_{1}, e_{1}\right) \\
& -g^{2}\left(J A e_{1}, e_{2}\right)+\frac{1}{2}\left\{\sum_{i \neq j}\left(h_{i j}^{n+1}\right)^{2}+\epsilon\right\}-\left(h_{12}^{n+1}\right)^{2}-g\left(\phi S e_{2}, e_{2}\right)-g\left(\phi S e_{1}, e_{1}\right) \\
& +\eta\left(e_{2}\right)^{2}+\eta\left(e_{1}\right)^{2} \\
= & 3 g^{2}\left(\phi e_{1}, e_{2}\right)+g\left(A e_{2}, e_{2}\right) g\left(A e_{1}, e_{1}\right)-g^{2}\left(A e_{1}, e_{2}\right)+g\left(J A e_{2}, e_{2}\right) g\left(J A e_{1}, e_{1}\right) \\
& -g^{2}\left(J A e_{1}, e_{2}\right)+\frac{\epsilon}{2}+\left(h_{12}^{n+1}\right)^{2}+\sum_{i \neq j, i, j \geq 2}\left(h_{i j}^{n+1}\right)^{2}-\left(h_{12}^{n+1}\right)^{2}-g\left(\phi S e_{2}, e_{2}\right) \\
& -g\left(\phi S e_{1}, e_{1}\right)+\eta\left(e_{2}\right)^{2}+\eta\left(e_{1}\right)^{2} \\
\geq & 3 g^{2}\left(\phi e_{1}, e_{2}\right)+g\left(A e_{2}, e_{2}\right) g\left(A e_{1}, e_{1}\right)-g^{2}\left(A e_{1}, e_{2}\right)+g\left(J A e_{2}, e_{2}\right) g\left(J A e_{1}, e_{1}\right) \\
& -g^{2}\left(J A e_{1}, e_{2}\right)-g\left(\phi S e_{2}, e_{2}\right)-g\left(\phi S e_{1}, e_{1}\right)+\eta\left(e_{2}\right)^{2}+\eta\left(e_{1}\right)^{2}+\tilde{\tau} \\
& -\frac{3(n-1)-2}{2}-\frac{g^{2}(A N, N)}{2}-\frac{n^{2}(n-2)}{2(n-1)}\|\tilde{\mathcal{H}}\|^{2}+(n-1) g\left(\phi S e_{i}, e_{i}\right)
\end{aligned}
$$

Thus, we derive

$$
\begin{aligned}
\tilde{\tau}(p)-\tilde{\mathcal{K}}(\pi) \leq & (n-2)\left\{\frac{n^{2}\|\tilde{\mathcal{H}}\|^{2}}{2(n-1)}+\frac{3(n-1)-2}{2(n-2)}\right\}-3 g^{2}\left(\phi e_{1}, e_{2}\right)-g\left(A e_{2}, e_{2}\right) g\left(A e_{1}, e_{1}\right) \\
& +g^{2}\left(A e_{1}, e_{2}\right)-g\left(J A e_{2}, e_{2}\right) g\left(J A e_{1}, e_{1}\right)+g^{2}\left(J A e_{1}, e_{2}\right)+g\left(\phi S e_{2}, e_{2}\right) \\
& +g\left(\phi S e_{1}, e_{1}\right)-\eta\left(e_{2}\right)^{2}-\eta\left(e_{1}\right)^{2}+\frac{g^{2}(A N, N)}{2}-(n-1) g\left(\left(\phi S e_{i}, e_{i}\right)\right.
\end{aligned}
$$


or, equivalently

$$
\begin{aligned}
\tilde{\tau}(p)-\tilde{\mathcal{K}}(\pi) \leq & (n-2)\left\{\frac{n^{2}\|\tilde{\mathcal{H}}\|^{2}}{2(n-1)}+\frac{3(n-1)-2}{2(n-2)}\right\}+g^{2}\left(A e_{1}, e_{2}\right)+g^{2}\left(J A e_{1}, e_{2}\right) \\
& +\frac{g^{2}(A N, N)}{2}+g\left(\phi S e_{1}, e_{1}\right)+g\left(\phi S e_{2}, e_{2}\right)+(n-1) g\left(\phi S e_{i}, e_{i}\right)
\end{aligned}
$$

Now, finally we get equality in (6.1) at $p \in \mathcal{M}$ if and only if we have the equality case of lemma.

$$
\begin{aligned}
h_{i j}^{n+1} & =0 \text { for all } i \neq j, \\
h_{11}^{n+1}+h_{22}^{n+1} & =h_{33}^{n+1}=h_{44}^{n+1}=\ldots=h_{n n}^{n+1} .
\end{aligned}
$$

Thus, we may have the choice for $\left\{e_{1}, e_{2}\right\}$ such that $h_{12}^{n+1}=0$. Hence, the matrix of the shape operator has the form (6.2).

Corollary 6.1. Let $\mathcal{M}$ be a real hypersurface of $Q^{m}$ admitting SSMC $\tilde{\nabla}$. Then, for a $\mathcal{U}$-principal normal vector field, we have

$$
\begin{aligned}
\tilde{\tau}(p)-\mathcal{K}(\pi) \leq & (n-2)\left\{\frac{n^{2}\|\tilde{\mathcal{H}}\|^{2}}{2(n-1)}+\frac{3(n-1)-1}{2(n-2)}\right\}+g^{2}\left(A e_{1}, e_{2}\right)+g^{2}\left(J A e_{1}, e_{2}\right) \\
& +g\left(\phi S e_{1}, e_{1}\right)+g\left(\phi S e_{2}, e_{2}\right)+(n-1) g\left(\phi S e_{i}, e_{i}\right),
\end{aligned}
$$

where $\pi \subset T_{p} \mathcal{M}$ is a 2-plane section spanned by tangent vectors $e_{1}$ and $e_{2}$.

Corollary 6.2. Let $\mathcal{M}$ be a real hypersurface of $Q^{m}$ admitting SSMC $\tilde{\nabla}$. Then, for a $\mathcal{U}$-isotropic normal vector field, we have

$$
\begin{aligned}
\tilde{\tau}(p)-\mathcal{K}(\pi) \leq & (n-2)\left\{\frac{n^{2}\|\tilde{\mathcal{H}}\|^{2}}{2(n-1)}+\frac{3(n-1)-2}{2(n-2)}\right\}+g^{2}\left(A e_{1}, e_{2}\right)+g^{2}\left(J A e_{1}, e_{2}\right) \\
& +g\left(\phi S e_{1}, e_{1}\right)+g\left(\phi S e_{2}, e_{2}\right)+(n-1) g\left(\phi S e_{i}, e_{i}\right),
\end{aligned}
$$

where $\pi \subset T_{p} \mathcal{M}$ is a 2-plane section spanned by tangent vectors $e_{1}$ and $e_{2}$.

\section{Acknowledgments}

Authors wishes to express sincere thanks to the referees for their valuable suggestions and comments towards the improvement of the paper.

\section{References}

[1] Arslan, K., Ezentas, R., Mihai, I., Özgür, C., Certain inequalities for submanifolds in $(k, \mu)$-contact space forms. Bull. Aust. Math. Soc., 64 (2001), no. 2, 201-212.

[2] Bansal, P., Shahid, M. H., Non-existence of Hopf real hypersurfaces in complex quadric with recurrent Ricci tensor, Appl. Appl. Math. 13 (2018), in press.

[3] Bansal, P., Shahid, M. H., Optimization approach for bounds involving generalised normalised $\delta$-Casorati curvatures, Advances in Intelligent Systems and Computing, 741 (2018), 227-237.

[4] Bansal, P., Shahid, M. H., Bounds of generalized normalized $\delta$-Casorati curvatures for real hypersurfaces in the complex quadric, Arab. J. Math., (2018), in press.

[5] Berndt, J., Suh, Y. J., Real hypersurfaces with isometric Reeb flow in complex quadrics, Internat. J. Math., 24 (2013), 1350050, 18pp.

[6] Blair, D. E., Contact manifolds in Riemannian Geometry. Lecture Notes in Math, 509, Springer-Verlag, Berlin, (1976).

[7] Chen, B. Y., Differential Geometry of Warped Product Manifolds and Submanifolds, World Scientific Publishing Co. Pte. Ltd., Hackensack, NJ, 2017.

[8] Chen, B. Y., Geometry of warped products as Riemannian submanifolds and related problem, Soochow J. Math., 28 (2002), 125-157.

[9] Chen, B. Y., Some pinching and classification theorems for minimal submanifolds, Arch. Math. (Basel), 60 (1993), 568-578.

[10] Chen, B. Y., A Riemannian invariant and its applications to submanifold theory, Result. Math., 27 (1995), 17-26.

[11] Chen, B. Y., Ideal Lagrangian immersions in complex space forms, Math. Proc. Cambridge Philos. Soc., 128 (2000), 511-533

[12] Chern, S. S., Minimal Submanifolds in a Riemannian Manifold, University of Kansas Press, (1968).

[13] Cioroboiu, D., Chen, B. Y., inequalities for semi-slant submanifolds in Sasakian space forms, Int. J. Math. Math. Sci, 27 (2003), $1731-1738$.

[14] Hayden, H. A., Subspaces of a space with torsion, Proc. Lond. Math. Soc., 34 (1932), 27-50. 
[15] Mihai, A., Özgür, C., Chen inequalities for submanifolds of real space forms with a semi-symmetric metric connection, Taizanese J. Math., 14 (2010), 1465-1477.

[16] Reckziegel, H., On the geometry of the complex quadric, in :Geometry and Topology of Submanifolds VIII (Brussels/Nordfjordeid 1995), World Sci. Publ., River Edge, NJ, (1995), 302-315.

[17] Suh, Y. J., Real hypersurfaces in the complex quadric with parallel Ricci tensor, Advances in Mathematics, 281 (2015), 886-905.

[18] Suh, Y. J., Real hypersurfaces in the complex quadric with Reeb parallel shape operator, Internat. J. Math., 25 (2014), 1450059, 17pp.

[19] Suh, Y. J., Psuedo-Einstein real hypersurfaces in the complex quadric, Math. Nachr., 290 (2017), no. 11-12, 1884-1904.

[20] Yano, K., On semi-symmetric metric connection, Rev. Roumaine Math. Pures Appl., 15 (1970), 1579-1586.

\section{Affiliations}

POOJA BANSAL

AdDRESS: Department of Mathematics, Faculty of Natural Sciences, Jamia Millia Islamia, New Delhi-110025, India.

E-MAIL: poojabansal811@gmail.com

ORCID ID : 0000-0002-5894-8027

\section{SIRAJ UDDIN}

ADDRESs: Department of Mathematics, Faculty of Science, King Abdulaziz University, 21589 Jeddah, Saudi Arabia.

E-MAIL: siraj.ch@gmail.com

ORCID ID : 0000-0002-3564-6405

MOHAMMAD HASAN SHAHID

ADDRESS: Department of Mathematics, Faculty of Natural Sciences, Jamia Millia Islamia, New Delhi-110025, India.

E-MAIL: hasan_jmi@yahoo.com

ORCID ID : 0000-0002-3646-4697 